\title{
CHANGES IN VISUAL FUNCTION WITH NORMAL AGEING, CATARACT AND INTRAOCULAR LENSES
}

\author{
J. D. MORRISON and J. L. JAY \\ Glasgow
}

\begin{abstract}
SUMMARY
We have been able to separate the neural and optical components of vision with contrast sensitivity tests employing laser interferometry and direct viewing of a cathode ray tube display. In normal ageing, neural function declined markedly while optical function remained essentially unchanged. On the other hand, even the mildest degree of cataract was shown to degrade optical function to the extent that it exceeded the age-related neural deterioration. The satisfactory optical performance of the monofocal implant lens has been confirmed, though the diffractive bifocal implant lens was shown to have optical limitations. A major cause of these was shown, by simulation experiments, to be the dilution of the contrast of the in-focus image by the superimposed defocused image, particularly under conditions of reduced retinal sensitivity as would be present in the elderly.
\end{abstract}

The decline in Snellen acuity above the sixth decade is extensively documented; ${ }^{1}$ however, while the Snellen test remains important for a rapid visual assessment, it provides only a single measurement of visual performance at the limit of resolution for the combined effects of the ocular media and retina/brain. Weatherill ${ }^{2}$ has highlighted the advantages of making measurements of contrast sensitivity in response to vertical sinusoidal grating patterns for a range of spatial frequencies which represent a range of different sizes of retinal images. Like the Snellen test, the results estimate the combined effects of the optical and neural components of the visual system. Weatherill has also described a refinement of the method which involves the projection of laser interference fringes directly onto the retina without undergoing refraction by the ocular media. These laser contrast sensitivity measurements are thus determined solely by the neural component of the visual system. Knowing the laser interferometric contrast

From: Institute of Physiology, University of Glasgow, Glasgow G12 8QQ, and Tennent Institute of Ophthalmology, Western Infirmary, Glasgow G11 6NT, UK.

Correspondence to: J. D. Morrison, Institute of Physiology, University of Glasgow, Glasgow G12 8QQ, UK. sensitivities, it then becomes possible to estimate the contribution of the ocular media as a separate function.

This entails calculation of the optical contrast ratio at each spatial frequency of sinusoidal grating pattern. The optical contrast ratio is the contrast sensitivity in response to the refracted cathode ray tube (CRT) display (i.e. optical $\times$ neural) divided by the contrast sensitivity in response to the laser interference fringes (i.e. neural). In the visual system of a normal individual the rate of decline of the optical contrast ratio with spatial frequency is considerably less than the rate of decline of the interferometric contrast sensitivity, which usually sets the limit of resolution at about $\left.55 \mathrm{c} / \mathrm{deg}^{3}{ }^{3}\right)$ Hence, determinations of the interferometric contrast sensitivity function and the optical contrast ratio function provide assessments of the neural function and optical function, respectively, of the visual system. The purpose of this contribution is to determine the ways in which the optical function of the eye might be affected by normal ageing, cataract formation and the presence of an intraocular lens.

\section{METHODS}

The apparatus employed in our experiments was assembled within our laboratory, although commercial apparatus is also available. Vertical sinusoidal grating patterns are generated on a Tektronix 606B monitor with a green phosphor, which due to its very small beam diameter allows the generation of a high spatial frequency display without diminution of contrast. ${ }^{4}$ This pattern may be modulated on and off or reversed instantaneously, according to the requirements of the experiment, while maintaining a constant overall luminance. The vertical interference fringes which are seen in the Maxwellian view through a microscope objective are generated from a red helium-neon laser after attenuation of intensity to match the luminance of the monitor. The interference fringes, which possess a sinusoidal intensity profile, are adjustable continuously in terms of contrast and spatial frequency. ${ }^{5}$

After the standard Snellen test and refraction appro- 
priate to the viewing distance, the subject undergoes a familiarisation procedure in which contrast threshold is determined 5-8 times for each of an abbreviated range of spatial frequencies. For both the CRT display and interferometer the subject slowly increases the contrast until the grating pattern is just visible and no more. It is emphasised that the overall luminance/intensity remains constant despite changing contrast. The value obtained is the contrast threshold, the reciprocal of which is the contrast sensitivity, which is usually expressed on a logarithmic scale due to the very wide range of values obtainable. This ascending method of threshold contrast determination has the advantage of being relatively rapid while at the same time giving results very similar to those obtained with a much more time-consuming protocol in which the contrast for $50 \%$ of seeing was determined. ${ }^{6}$

It is possible to measure the contrast sensitivities for an extended range of spatial frequencies which may take, at a measured pace so as not to fatigue the subject, some 2-3 hours for each set-up. In practice we find that it is a pleasure to work with our older subjects, who wholeheartedly give of their best; the youngest subjects, however, may be somewhat restive as the day draws on. For each spatial frequency the mean contrast threshold and, hence, the mean contrast sensitivity is calculated. For clarity, the scatter of the individual readings in Figs. 1 and 3-6 has been omitted, but usually has a standard deviation of less than \pm 0.2 logarithmic units.

As described earlier, the optical contrast ratio is the quotient of the CRT contrast sensitivity divided by the interferometric contrast sensitivity. This value is always less than 1.00 due to degradation of contrast by the ocular media of the eye. For example, if the CRT contrast sensitivity at $10 \mathrm{c} / \mathrm{deg}$ were 1.0 logarithmic units and the interferometric contrast sensitivity were 1.3 logarithmic units, the optical contrast ratio would be -0.3 logarithmic units, i.e. 0.5 , indicating that $50 \%$ of the contrast had been lost on transmission by the ocular media. In practice, undue importance should not be assigned to the absolute value of the optical contrast ratio, which may sometimes be affected by inter-subject differences in viewing the two types of display. (While it would be desirable to employ two displays of the same wavelength, and indeed this is now possible with the advent of green helium-neon lasers, the difference in wavelength has been shown to make no appreciable difference to the contrast sensitivity. ${ }^{3}$ ) The important comparison is between the rate of decline of the optical contrast ratio with spatial frequency compared with the rate of decline of the interferometric contrast sensitivity with spatial frequency. If the optical contrast function declines faster than the interferometric contrast sensitivity function then the ocular media must be limiting visual resolution.

\section{NORMAL AGEING}

Numerous studies have confirmed the loss of contrast sensitivity over an extended range of spatial frequencies during the lifespan..$^{7-10}$ This would imply an impairment in the appreciation of all the levels of spatial detail which make up the visual image. The loss at medium and high spatial frequencies is illustrated in the comparison of CRT data between a 34-year-old subject and a 78-year-old subject, in which contrast sensitivities were depressed in the older person by some 0.4-0.6 logarithmic units (Fig. 1, open and filled circles). This reflects the trends shown in the group data from 45 subjects aged 15-86 years which are presented for the different decades (Fig. 2A). ${ }^{5}$ To go beyond the stage of simply recording the ageing change to that of identifying its major cause requires a systematic investigation of the component stages. For instance, adoption of a signal detection protocol, which assesses the criterion of a subject's judgement as well as his or her threshold, has revealed there to be no significant impairment of decision-making by the elderly. ${ }^{6}$ This gives confidence that the age-related loss of contrast sensitivity has its origins in the conventionally defined visual system rather than in the higher reaches of the cerebral cortex.

It is also apparent from the comparisons between the young and elderly subjects that the retinal contrast sensitivities in response to the non-refracted laser interference fringes were also reduced with age (Fig. 1, open and filled triangles). The slope values appended to Fig. 1 indicate a steepening of the fall-off in interferometric contrast sensitivity with increasing spatial frequency in the older sub-

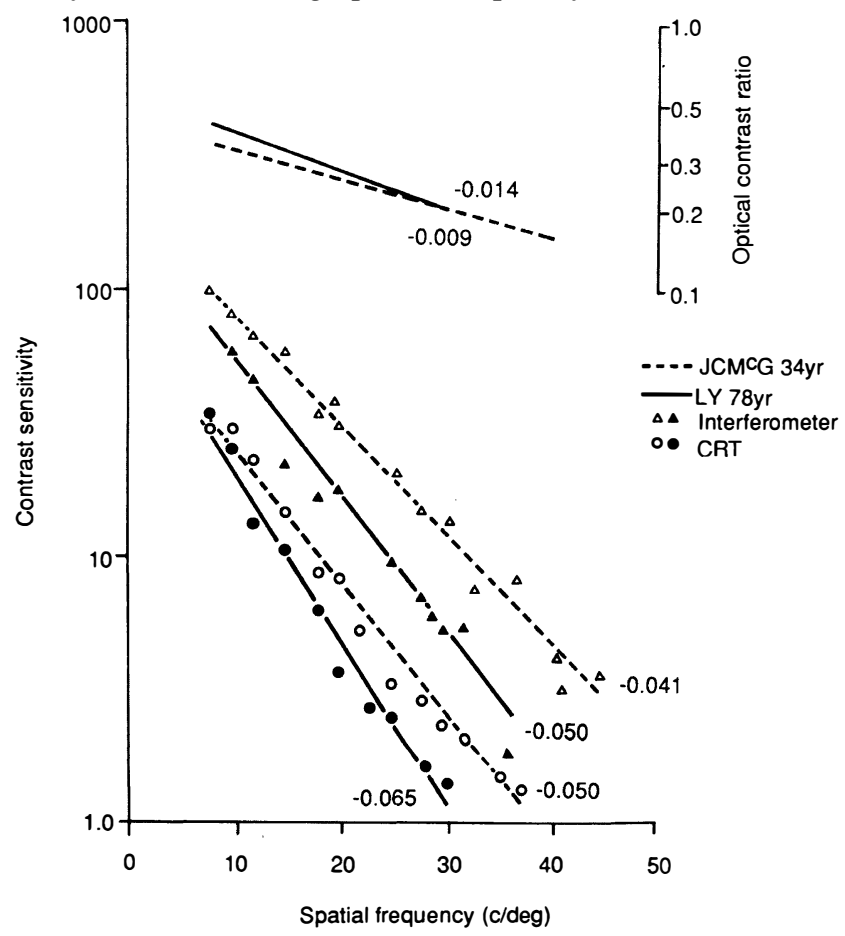

Fig. 1. The fall in contrast sensitivities for uniocular viewing in response to sinusoidal grating patterns generated by CRT and laser interferometer for a normal elderly subject (filled symbols) compared with a normal young subject (open symbols). The numerical value appended to each line is the slope of the line derived by linear regression analysis. Each point is the mean of $5-8$ determinations with a standard deviation of less than \pm 0.2 . The optical contrast ratio is derived as the quotient of CRT and interferometer contrast sensitivities. Note that while a logarithmic scale compresses the range of data greater than 1, it serves to accentuate the spread for the optical contrast ratios. 


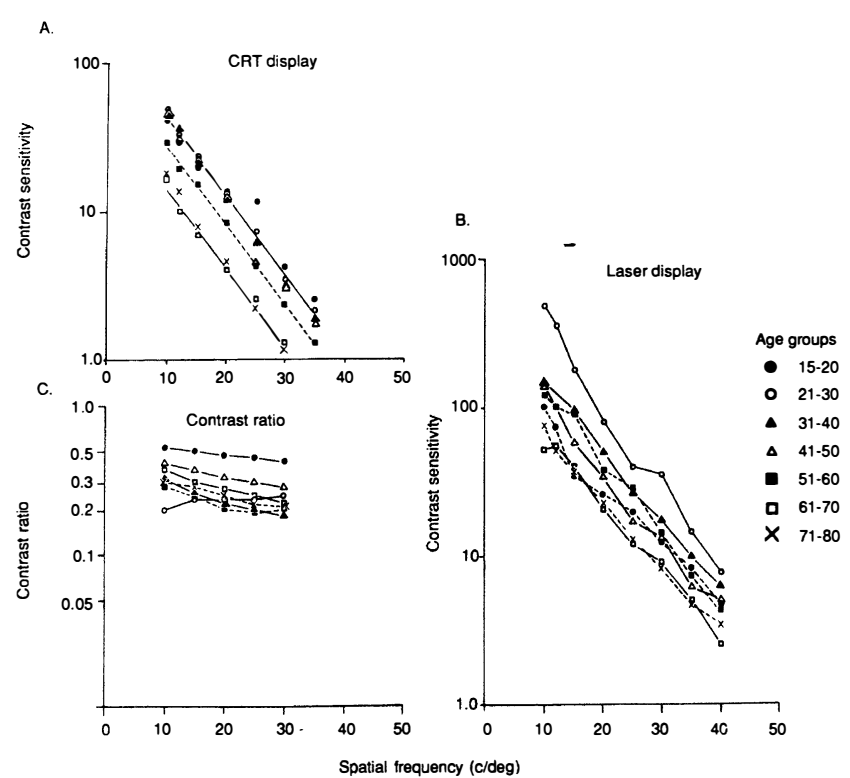

Fig. 2. The age-related decline in contrast sensitivities for the $C R T$ display $(A)$ and laser display $(B)$, and the constancy of the calculated optical contrast ratios $(C)$ arranged by decade for different spatial frequencies. (From Morrison and McGrath. ${ }^{5}$ )

ject. When the optical contrast ratio function was derived by subtraction of the regression line for the interferometric data from that for the CRT data, it was very similar in the young and elderly subjects as shown by similar slope values (Fig. 1, top panel). Thus, in both subjects, transmission of contrast by the ocular media was not the ultimate factor in limiting resolution but, rather, the major contribution arose from the deterioration of the interferometric contrast sensitivities determined by the retina/ brain. This is shown more comprehensively by the group data, in which the interferometric contrast sensitivities declined markedly with increasing age at all the spatial frequencies studied (Fig. 2B) while the optical contrast ratio showed a much smaller change (Fig. 2C). Thus, these results indicate that the age-related loss in vision is primarily neural in origin. This conclusion has subsequently been supported by several independent studies involving interferometric measurements, ${ }^{11}$ measurement of displacement threshold hyperacuity, ${ }^{12}$ and visual assessment of patients with acrylic intraocular lenses. ${ }^{13,14}$

\section{CATARACT}

The measurement of CRT contrast sensitivities in cataract patients has identified two groups which may represent different stages of cataract development. ${ }^{15}$ One group showed a loss of contrast sensitivity at medium and high spatial frequencies but not at low spatial frequencies, whereas the other group showed a loss of contrast sensitivity at all spatial frequencies. This latter group would be particularly disadvantaged visually since low spatial frequency vision is essential for the appreciation of the overall form of a visual scene. There was no correlation between the nature of the cataract, i.e. whether cortical or nuclear, and the type of contrast sensitivity loss. Rather, the overall spatial frequency loss tended to arise after the lens opacity had become uniform, when wide angle light scattering caused a loss of contrast sensitivity at all spatial frequencies. The earlier stage was suggested to be dominated by localised optical aberrations exerting an effect primarily at the high spatial frequencies. Thus, while Snellen acuity measurements might be similar in the two groups, only contrast sensitivity measurements at low spatial frequencies would serve to quantitate the seriousness of the visual impairment experienced by the patient.

The profound consequences on the CRT contrast sensitivities of very early diffuse cortical and nuclear opacities in one eye of an elderly man, compared with the companion eye which contained a monofocal implant following extracapsular extraction, are shown in Fig. 3 (open and filled circles, respectively). Resolution for the cataractous eye was at best $6 \mathrm{c} / \mathrm{deg}$ compared with $21 \mathrm{c} / \mathrm{deg}$ for the monofocal implant eye, while Snellen acuities after refraction were $6 / 18$ and $6 / 9$, respectively. In this case, the presence of the diffuse cataract also created some difficulties in detecting the laser interference fringes, so that the interferometric contast sensitivities were appreciably reduced compared with the data for the monofocal implant eye (Fig. 3, open and filled triangles). This would cause an overestimation of the optical contrast ratio. While some qualification must be attached to the optical contrast transmission results calculated for the cataractous eye, a negative slope of almost $\times 3$ that for the monofocal implant eye conveys the considerable impairment of ocular quality caused even by such an early cataract.

Data in Fig. 4 from an 81-year-old woman, on the other hand, show very good CRT contrast sensitivities for both

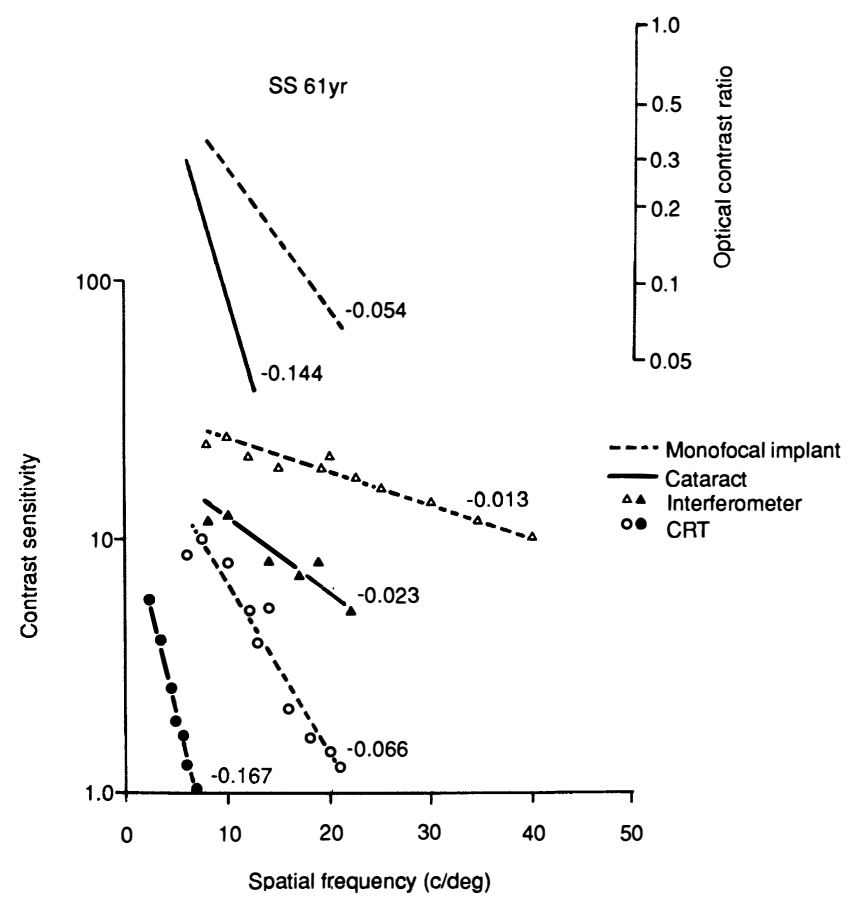

Fig. 3. The adverse performance of the cataractous eye in terms of CRT contrast sensitivities and optical function (filled symbols) compared with the monofocal implant eye (open symbols) of an elderly male patient. For further details see legend to Fig. 1 . 


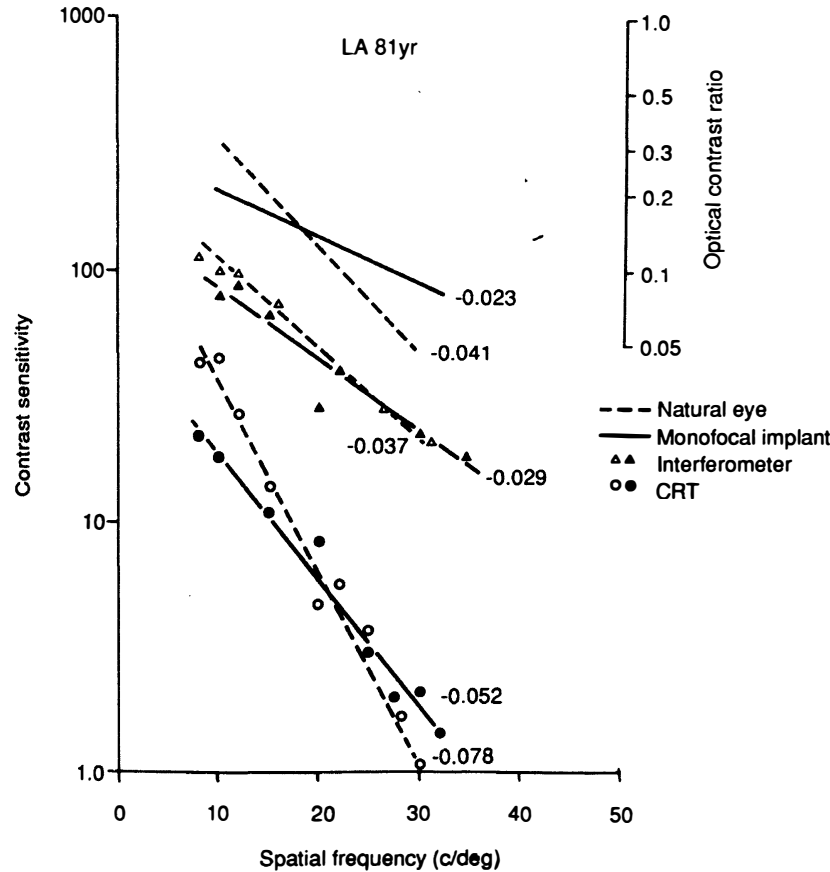

Fig. 4. The slight impairment of CRT contrast sensitivities and optical function by the natural eye containing a very mild cataract (open symbols) compared with the monofocal implant eye (filled symbols) of an elderly female patient.

her monofocal implant eye (filled symbols) and her companion eye which was described as having very early senile nuclear sclerosis with some minor cortical spoke opacities (open symbols). Both eyes had a Snellen acuity of 6/9 after refraction. The interferometric contrast sensitivities are very similar for the two eyes (filled and open triangles).

Derivation of the optical contrast transmission line reveals it to be appreciably steeper for the natural eye (slope $m=-0.041$ ) than for the monofocal implant eye $(m=-0.023)$. Of particular relevance is the fact that the optical contrast transmission in the natural eye declines slightly more steeply with increasing spatial frequency $(m=-0.041)$ than do the interferometric contrast sensitivities $(m=-0.037)$, so much so that the ocular media may be concluded to be now contributing appreciably to the limitation of spatial resolution. This is a departure from the data for young subjects and, indeed, from the data for that patient's monofocal implant eye, which indicates that the cataract, although described as very mild indeed, was adversely affecting the patient's spatial vision.

These examples, in addition to providing an assessment of the quality of ocular function, show that neural function was also essentially satisfactory. However, laser interferometry is of limited value when there is more than the most minor degree of opacity which would markedly degrade the laser interference pattern. Nevertheless, laser interferometry stills retains an advantage over modern ophthalmoscopy in cases of minor opacities as it can reveal the presence of amblyopia or other conditions with a normal fundus appearance.

\section{MONOFOCAL IMPLANTS}

Insertion of a monofocal acrylic lens implant following extracapsular cataract extraction results in an improvement in colour perception due to reduced chromatic aberration, in addition to improved transmission (especially at shorter wavelengths) due to removal of the elderly lens' yellow-brown pigment, ${ }^{16}$ and in improved CRT contrast sensitivities. ${ }^{17}$ The visual performance for the monofocal implant eye of the 81-year-old patient shown in Fig. 4 is well above average for the norm for that age group. The optical contrast transmission (continuous line) shows a less steep fall-off with spatial frequency $(m=-0.023)$ than the interferometric contrast sensitivity $(m=-0.029)$ (filled triangles), indicating its satisfactory performance. Another example from this series is shown elsewhere. ${ }^{5}$ However, visual performance for the implant eye is not always satisfactory. ${ }^{18}$ Occasionally, the patient has subnormal vision for no apparent reason.

In Fig. 5A (open circles) the CRT contrast sensitivities for an elderly woman with a monofocal implant giving a Snellen acuity after refraction of $6 / 12$ were below expectation compared with the norm for that age group. ${ }^{18}$ This was attributed to slight haziness of the posterior capsule though, after division of the capsule by YAG laser capsulotomy, no improvement in contrast sensitivity was effected (Fig. 5A, crosses). The interferometric contrast sensitivities were essentially normal, indicating that her poor vision was not attributable to, say, amblyopia. In fact, this patient should have had CRT contrast sensitivities similar to those shown in Fig. 5B (open circles) or in
A

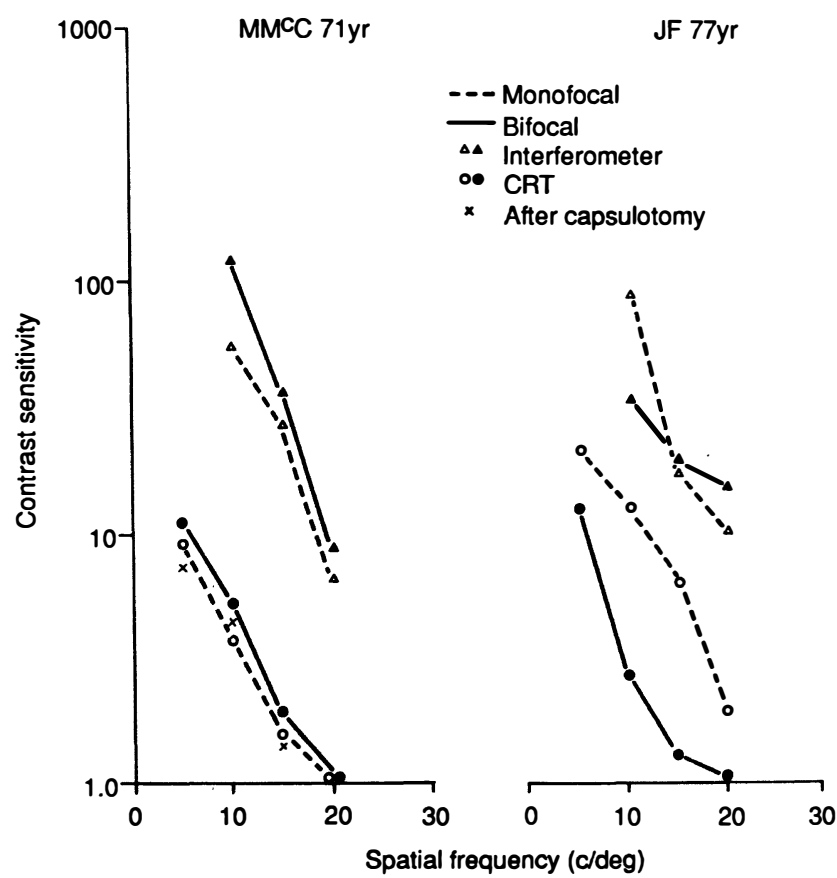

Fig. 5. The limited performance of the diffractive bifocal implant eye in terms of CRT contrast sensitivities (filled symbols) for two elderly female patients also possessing a monofocal implant eye (open symbols). In (A), additional data after YAG laser capsulotomy (crosses) are shown. 
Fig. 1 (filled circles). Her poor vision must thus be attributable to optical factors though, given the absence of improvement after capsulotomy, an explanation for the anomaly is not readily apparent. On the other hand, the patient shown in Fig. 5B had interferometric and CRT contrast sensitivities that were both normal for her age (Snellen acuity 6/5), indicating the satisfactory performance of the monofocal implant.

\section{BIFOCAL IMPLANTS}

The 3M diffractive bifocal intraocular lens offers an enhanced depth of focus, apparently without the problem of centration associated with two-zone intraocular lenses. ${ }^{19}$ However, evaluation of distance vision and near vision of patients. with this type of lens indicates some compromise on the clarity of vision. ${ }^{20,21}$ In an in-depth study of two patients with a diffractive bifocal lens in one eye and a monofocal lens in the other for comparison, it was shown that the bifocal lens did indeed confer a greater depth of focus compared with the monofocal eye. ${ }^{18}$ However, in both patients the CRT contrast sensitivities were appreciably less than would have been expected from the interferometric contrast sensitivities (which were within the normal range), thus again excluding the possibility of amblyopia. In one patient the CRT contrast sensitivities were similar to those for her anomalous monofocal eye (Fig. 5A), while for the other patient they were appreciably poorer than for her monofocal eye (Fig. 5B). This indicates that the increased depth of focus was acquired at the expense of spatial resolution.

\section{SIMULATION EXPERIMENTS}

The inference that the reduced retinal illumination of some 0.5 logarithmic units, caused by senile miosis and reduced lenticular transmission, is not a major factor in the age-related loss in vision ${ }^{22}$ has been confirmed experimentally. CRT contrast sensitivities in young subjects remained unaffected while wearing an artificial pupil or 0.5 logarithmic unit neutral density filter. ${ }^{5}$ The former condition has subsequently been confirmed, ${ }^{23,24}$ while the effects of progressive reductions in illumination are shown in Fig. 6A. At the spatial frequency of $5 \mathrm{c} / \mathrm{deg}$, CRT contrast sensitivity was unaffected by a 0.3 logarithmic attenuation in a young subject. Further attenuations produced a progressive fall, though the contrast sensitivity never descended to those of the two elderly patients. It was notable that the 0.3 logarithmic unit attenuation always caused a fall in contrast sensitivity in the two elderly subjects. This greater susceptibility to reduced retinal illumination is undoubtedly due to the reduced visual sensitivity to illumination, ${ }^{25}$ which is attributable to diminished availability of visual pigments in the photoreceptors. ${ }^{26,27}$ However, even when young and old subjects are placed on an equal basis in terms of pupil diameter and photopic threshold to light, contrast sensitivities still show a decline with increasing age (Muir, Barlow and Morrison, unpublished data).

In assessing the possible reasons for the impaired per- formance of the bifocal implant eyes of the two patients in Fig. 5, contrast sensitivities were measured in young subjects in response to two exactly superimposed CRT displays combined through a beam splitter. ${ }^{18}$ These data are shown in Fig. 6B (open circles). One CRT display was defocused by a positive lens calculated to give a net defocus of +3.64 DS, i.e. similar to the +3.5 DS conferred by the diffractive effects of the $3 \mathrm{M}$ bifocal lens, with the same image magnification. ${ }^{18}$ In this case, contrast sensitivities were consistently reduced by some $28 \%$ (Fig. $6 \mathrm{~B}$, filled circles). When a uniform background was substituted for the defocused display, which would theoretically reduce the display contrast by $50 \%$, a further decrement in contrast sensitivities occurred (triangles) to give a total reduction from the control values (open circles) of $53 \%$. This basically reveals the theoretical impossibility of optimal spatial resolution when there is superimposition of a defocused image on the in-focus image, thus resulting in diminished contrast of the in-focus image. Of particular importance was the finding that this result was obtained for the same effective retinal illumination as would be experienced by the elderly patients. If the young subjects carried out the experiment described in Fig. 6B without attenuation of the display luminance which had previously been employed to place them on a par with the older subjects, a reduction in contrast sensitivities with the defocused display superimposed upon the in-focus display was not detected. ${ }^{18}$ This indicates that the superimposed defocused image is tolerated appreciably better when levels of retinal illumination are sufficiently high, which would normally be the case in young subjects in daylight. A possible reduction in visual function in twilight conditions even for young patients deserves further study.

An evaluation of the diffractive bifocal intraocular lens, therefore, must consider the following points: (1) The div-

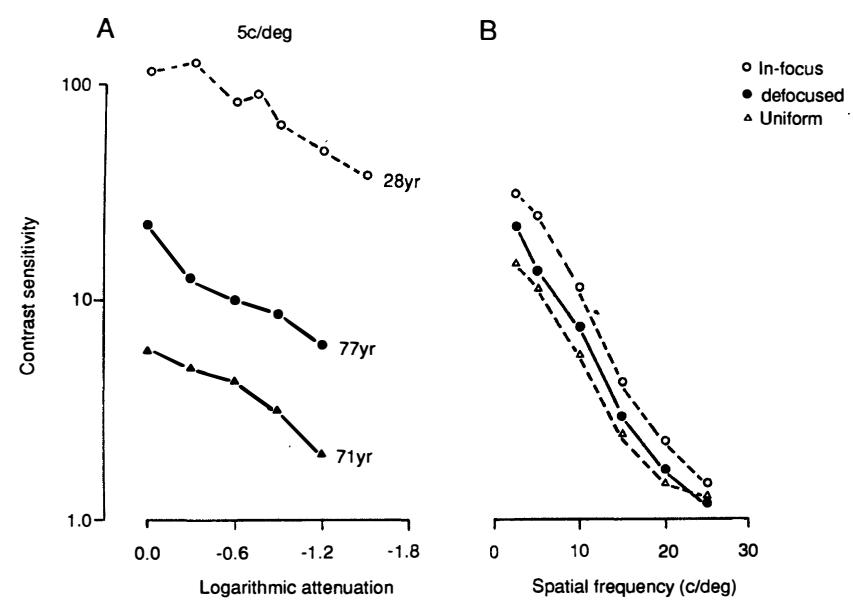

Fig. 6. (A) Adverse effects of luminance attentuation on CRT contrast sensitivity at 5 cldeg for one young and two elderly subjects. (B) Simulation of diffractive bifocal intraocular implant by simultaneous viewing of two accurately aligned CRT displays with: both displays in-focus (open circles), one display defocused by the equivalent of $+3.64 \mathrm{DS}$ at the nodal point of the eye (filled circles), and one display acting as a uniform background (triangles). (For further details of the experimental apparatus see Jay et al. ${ }^{18}$ ) 
ision of the available light between two foci may be critical in the elderly, whose retinal sensitivity to light is reduced. (2) Dilution of contrast of the in-focus image by superimposition of the defocused image, especially within the range of retinal sensitivities present in the elderly. (3) The unknown effect of a possible phase shift, i.e. a lateral shift of the defocused image with respect to the in-focus image, producing destructive interference which would cause an additional deleterious effect on contrast sensitivity. ${ }^{18}$ It would therefore seem essential to assess the requirements of a potential recipient of an intraocular lens, i.e. whether optimal acuity or increased depth of focus was all important, before recommending either the monofocal or diffractive bifocal lens.

\section{CONCLUSIONS}

Measurements of contrast sensitivities in response to both CRT and laser interference fringe displays give a greater insight into visual function than could be provided by simpler assessments of visual function. Of particular value are the interferometric contrast sensitivities which reflect neural function and the derivation of the optical transmission function. While optical function has been shown to have deteriorated sufficiently so as to limit visual resolution in even very early cataract, for more severe cases there seems little new information to be gained other than what could otherwise be obtained with a thorough ophthalmoscopic examination coupled with the Snellen test. However, in patients with intraocular lens implants where the ocular media do not degrade the transmission of the visual stimuli, far more rigorous information is obtainable. The high quality of transmission by monofocal implants has been confirmed, though the occasional problem of anomalous substandard vision with these implants is plainly a case for more extensive investigation. The diffractive bifocal implant, on the other hand, has been shown to have optical limitations, and only by clear delineation of the limits of its performance can the most appropriate circumstances for its use be identified.

Key words: Ageing, Cataract, Contrast sensitivity, Intraocular lenses.

\section{REFERENCES}

1. Weale RA. Biography of the eye. London: HK Lewis, 1982. 2. Weatherill J. Visual acuity assessment. Eye 1993;7:26-8.

3. Campbell FW, Green DG. Optical and retinal factors affecting visual resolution. J Physiol (Lond) 1965;181:576-93.

4. Kay CD, Morrison JD. The effects of a single intramuscular injection of atropine sulphate on visual performance in man. Hum Toxicol 1987;6:165-72.

5. Morrison JD, McGrath C. Assessment of the optical contributions to the age-related deterioration in vision. Q J Exp Physiol 1985;70:249-69.
6. Morrison JD, Reilly J. An assessment of decision-making as a possible factor in the age-related loss in contrast sensitivity. Perception 1986;15:541-52.

7. McGrath C, Morrison JD. The effects of age on spatial frequency perception in human subjects. Q J Exp Physiol 1981; 66:253-61.

8. Derefeldt G, Lennerstrand G, Lundh B. Age variations in normal human contrast sensitivity. Acta Ophthalmol (Copenh) 1979;75:679-90.

9. Owsley C, Sekuler R, Siemsen D. Contrast sensitivity throughout adulthood. Vision Res 1983;23:689-99.

10. Ross JE, Clarke DD, Bron AJ. Effect of age on contrast sensitivity function: uniocular and binocular findings. $\mathrm{Br} \mathrm{J}$ Ophthalmol 1985;69:51-6.

11. Elliot DB. Contrast sensitivity decline with ageing: a neural or optical phenomenon? Ophthal Physiol Opt 1987;7: 415-9.

12. Elliot DB, Whitaker D, Thompson P. Use of displacement threshold hyperacuity to isolate the neural component of senile vision loss. Appl Optics 1989;28:1914-8.

13. Owsley C, Gardner T, Sekuler R, Lieberman H. Role of the crystalline lens in the spatial vision loss of the elderly. Invest Ophthalmol Vis Sci 1985;26:1165-70.

14. Jay JL, Mammo RB, Allan D. Effect of age on visual acuity after cataract extraction. Br J Ophthalmol 1987;71:112-5.

15. Hess R, Woo G. Vision through cataracts. Invest Ophthalmol Vis Sci 1978;17:428-35.

16. Jay JL, Gautam VB, Allan B. Colour perception in pseudophakia. Br J Ophthalmol 1982;66:658-62.

17. Weatherill J, Yapp M. Contrast sensitivity in pseudophakia and aphakia. Ophthalmic Physiol Opt 1986;6:297-301.

18. Jay JL, Chakrabarti HS, Morrison JD. Quality of vision through diffractive bifocal intraocular lenses. Br J Ophthalmol 1991;75:359-66.

19. Simpson MJ. The diffractive multifocal intraocular lens. Eur J Implant Refract Surg 1989;1:115-21.

20. Percival SPB. Prospective study of the new diffractive bifocal intraocular lens. Eye 1989;3:571-5.

21. Percival SPB, Setty SS. Comparative analysis of three prospective trials of multifocal implants. Eye 1991;5:712-6.

22. Weale RA. Senile changes in visual acuity. Trans Ophthalmol Soc UK 1975;95:36-8.

23. Kay CD, Morrison JD. A quantitative investigation into the effects of pupil diameter and defocus on contrast sensitivity for an extended range of spatial frequencies in natural and homatropinized eyes. Ophthalmic Physiol Opt 1987;7: 21-30.

24. Tobimatsu S, Celesia GG, Cone SB. Effects of pupil diameter and luminance changes on pattern electroretinograms and visual evoked potentials. Clin Vision Sci 1988;2: 293-302.

25. Gunkel RD, Gouras P. Changes in scotopic visibility thresholds with age. Arch Ophthalmol 1963;69:4-9.

26. Kilbride PE, Hutman LP, Fishman M, Read JS. Foveal cone pigment density difference in the aging human eye. Vision Res 1986;26:321-5.

27. Keunen JEE, Van Norren D, Van Meel GJ. Density of foveal cone pigment at older age. Invest Ophthalmol 1987;28: 985-91. 